\title{
ANÁLISIS DE LA SUSPENSIÓN INDEPENDIENTE SLA POSTERIOR PARA AUTOS DE COMPETICIÓN
}

\section{ANALYSIS OF THE INDEPENDENT SUSPENSION SLA POSTERIOR FOR CARS OF COMPETITION}

\author{
Phd. Albert Miyer Suarez Castrillon ${ }^{\mathrm{a}}$, MSc. Fabian Andres Fuentes Martinez ${ }^{\mathrm{b}}$, Phd. Sir Alexci \\ Suarez Castrillon ${ }^{\mathrm{c}}$ \\ ${ }^{a}$ Universidad de Pamplona, Investigador Grupo GIMUP. Km 1 vía Bucaramanga, Pamplona, \\ Norte de Santander, Colombia, (57+7)5685303. albertmiyer@unipamplona.edu.co \\ ${ }^{\text {b}}$ Universidad de Pamplona, Investigador Grupo GIMUP. Km 1 vía Bucaramanga, Pamplona, \\ Norte de Santander, Colombia, (57+7)5685303. \\ ${ }^{c}$ Universidad Francisco de Paula Santander Ocaña, Grupo de investigación GRUCITE \\ Via Acolsure - Sede el Algodonal, Ocaña, Colombia, sasuarezc@ufpso.edu.co
}

Fecha de recepción: 11-06-2016

Fecha de aprobación: 02-12-2016

Resumen: La finalidad de este estudio, es simular y analizar el diseño de una suspensión posterior de vehículos de competición, de la Serie NASCAR Sprint Cup, en cuanto a la configuración y reducción del peso a través de la utilización de herramientas computacionales, para lo cual se emplearan datos obtenidos de las bases de datos oficiales, para conocer todas las condiciones que debe cumplir el chasis, para de estar manera estudiar la dinámica del auto (transferencia lateral y longitudinal de carga incidente sobre la suspensión posterior), donde se calcularan las cargas a las que es sometida la suspensión.

Para la evaluación de los factores dinámicos del auto (condiciones de operación, fuerzas G's), se empleara el software CATIA V5R20 donde se realizará el modelo 3D de la suspensión para estudiar la cinemática (geometría) del mecanismo para analizar su comportamiento, de esta forma también mediante el uso del software se revisará cada uno los datos obtenidos en la fase de estudio y cálculo.

Palabras clave: SLA, Suspensión, Independiente, Brazos de Control, Reglajes. 


\begin{abstract}
The purpose of this study is to simulate and analyze the design of a rear suspension of competition vehicles, NASCAR Sprint Cup Series, in this way is intended to give a better choice of suspension system of these cars, in terms of the configuration And weight reduction through the use of computational tools, using data obtained from the official databases, to know all the conditions that the chassis must meet, so as to be able to study the dynamics of the car (lateral transfer And longitudinal load incident on the rear suspension), where the loads to which the suspension is subjected.

For the evaluation of the dynamic factors of the car (operating conditions, forces $G \mathrm{~s}$ ), the software CATIA V5R20 will be used where the 3D model of the suspension will be realized to study the kinematics (geometry) of the mechanism to analyze its behavior, of this So also through the use of the software will be reviewed each the data obtained in the study and calculation phase.
\end{abstract}

Keywords: SLA, Suspension, Independent Control Arms, adjustment.

\section{INTRODUCCIÓN}

La suspensión de los autos que corren en la National Association For Stock Auto Racing (NASCAR), deben ofrecer una gran estabilidad para proporcionándole al piloto una óptima maniobrabilidad a altas velocidades. hoy en día estos autos emplean suspensiones de eje rígido de tirantes en la parte posterior, que es un tipo de suspensión que limita su ajuste, impidiendo la configuración de la suspensión de acuerdo a las condiciones de la pista y comprometiendo así el rendimiento del auto, por lo que los equipos dependiendo del tipo de la pista emplean variaciones de los componentes que conforman la suspensión. Este estudio tiene como finalidad: analizar el diseño de una suspensión independiente; teniendo en cuenta la reducción del peso global del sistema y versatilidad a la hora de configurarla, por lo que se emplearán disciplinas referentes a la dinámica automotriz donde se estudiarán cada una de las cargas involucradas, el análisis cinemático proporcionará la geometría adecuada de los componentes de la suspensión de acuerdo a las cargas calculadas y al análisis estructural usando la herramienta del método de los elementos finitos (FEM) incorporada en el software, donde se evalúa el comportamiento de los componentes.

Las suspensiones que utilizan los vehículos que compiten en la serie NASCAR, emplean un sistema de suspensión no independiente en la parte posterior, conocida con el nombre de suspensión de eje rígido de tirantes, la cual es poco favorable en el tema de configuración (alineación), lo que obliga a las escuderías a reemplazar los componentes de la suspensión como tirantes, amortiguadores, resortes y barras panhards, por otro lado el sistema de frenos también es modificado debido a que existe varias opciones de mordazas y rotores que se emplean de acuerdo a la pista donde se vaya a competir.

El antiguo sistema de suspensión de eje rígido, no es una muy buena elección para 
autos de alto rendimiento ya que su alineación es limitada, de acuerdo con las características de las pistas este sistema permite alterar el comportamiento del auto antes y durante la carrera mediante un tornillo en el amortiguador posterior izquierdo de la suspensión (lo que permite alterar el cruce de peso), el estudio tiene como finalidad el análisis del diseño de una suspensión independiente

\section{METODOLOGÍA}

Para el desarrollo del proyecto se analizó la información pertinente sobre sistemas de suspensión con el fin de conocer las ventajas y desventajas de los diferentes sistemas, una vez terminada la fase de estudio de la información, se realizó el análisis matemático de la geometría para definir la posición de los componentes que conforman el sistema y posteriormente se realizo un análisis empleando el software CATIA V5R20 para evaluar la cinemática del sistema y el comportamiento mecánico de cada uno de los componentes.

\section{INTRODUCCIÓN A LOS SISTEMAS DE SUSPENSIÓN}

El sistema de suspensión de un auto tiene como misión mantener el contacto entre la rueda y la superficie de carrera para absorbiendo las irregularidades de la pista, con la finalidad de conseguir un mayor control a altas velocidades. La suspensión y la dirección son sistemas básicos e interrelacionados que conectan las ruedas al chasis.

Aun cuando los sistemas pueden variar en diseño, están construidos de manera que el auto pueda ser tripulado y dirigido con suavidad en una diversidad de condiciones de operación. Una suspensión debe tener dos cualidades; a)Elasticidad: para evitar golpes secos en el chasis debido a las irregularidades que pueda tener la pista y b) Amortiguación: que impida un excesivo balanceo de los elementos que constituyen la suspensión. Los sistemas de suspensión constan de tres componentes mecánicos, cuya función principal es la almacenar y absorber energía. Estas dos funciones las realizan normalmente dos componentes distintos: los elementos elásticos, comúnmente llamados resortes, y los elementos disipadores llamados amortiguadores.

\section{ELEMENTOS ELASTICOS}

- Los Resortes: proporcionan elasticidad en el movimiento hacia arriba o hacia abajo entre las ruedas y el chasis.

- Los amortiguadores: controlan o amortiguan la oscilación o acción de rebote de los resortes.

- Los componentes de conexión: estos serían los ejes, brazos, rótulas, pivotes, bujes, barras, etc.

\section{ELEMENTOS DISIPADORES}

Con respecto a la suspensión, el peso del auto se divide en dos partes:

- La masa suspendida: que comprende todos los elementos cuyo peso es soportado por el chasis (motor, tren de potencia, caja de cambios, etc.).

- La masa no suspendida, hace referencia al $50 \%$ del peso de los componentes que 
se conectan al auto como son los brazos de la dirección, brazos de control, manguetas, discos de freno, etc. es la parte del auto que está permanentemente en contacto con la superficie de carrera del circuito. El mantenimiento adecuado de los sistemas de suspensión es esencial tanto para el rendimiento del auto como para la seguridad de sus pasajeros.

\section{DEFINICIÓN DE LA GEOMETRÍA DE LA SUSPENSION}

Cuando se habla de la geometría, se hace referencia a la masa no suspendida del auto que está conectada a la masa suspendida, estas conexiones no solo describen la trayectoria de movimiento relativo, sino que también controlan las fuerzas que son transmitidas entre ellos. Para el desarrollo del análisis de la geometría de la suspensión SLA, es necesario analizar las trayectorias que describen los brazos de control, para ello se asume la longitud de los brazos de control tanto superior como inferior junto con la longitud del montante o mangueta, dado que son los parámetros seleccionados a priori para dicho análisis; esto se debe a que no existe una metodología que permita definir dichos parámetros con exactitud, por lo que hay que recurrir a suposiciones o buscar la solución mediante un método gráfico. En la figura 1 se describen las dimensiones de los brazos de control inferior y superior tomadas a priori.

Figura 1. Descripción de la ecuación del movimiento de la rueda

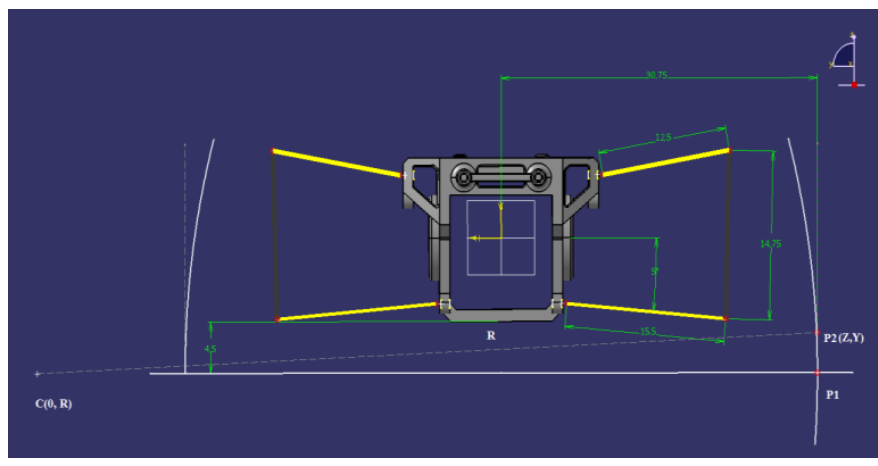

Fuente: Autores

A continuación se relaciona las ecuaciones de movimiento para la trayectoria que sigue la rueda, asumiendo que la trayectoria es como se muestra en la figura 5, dicha trayectoria curva tiene centro en las coordenadas $\mathrm{C}(0, \mathrm{R})$. Ahora se evalúa el recorrido que hace la rueda del punto $\mathrm{P} 1$ con coordenadas $(0, \mathrm{R})$ moviéndose por la trayectoria del círculo al punto $\mathrm{P} 2$ con coordenadas $(\mathrm{Z}, \mathrm{Y})$ en la dirección de $\mathrm{Z}$ y $\mathrm{Y}$, con el fin de plantear la ecuación de su movimiento.

$\mathrm{D}(\mathrm{P} 2, \mathrm{C})=\mathrm{R}$

Donde $\mathrm{R}$ es el radio de la circunferencia de la trayectoria que describe la rueda, con dicho radio se determina la distancia entre el punto P2 que recorre la suspensión y el centro de su trayectoria, teniendo en cuenta las coordenadas descritas anteriormente.

$\sqrt{(\mathrm{Z}-0)^{2}(\mathrm{Y}-\mathrm{R})^{2}}=\mathrm{R}$

$Z^{2} \pm(Y-R)^{2}=\mathrm{R}^{2}$ 
Mediante la relación planteada se obtiene la ecuación 1, la cual describe la trayectoria de la rueda dada una entrada en el eje $\mathrm{Z}$.

$$
Z^{2}=(2 . Y . R)-Y^{2}
$$

\section{CONSTRUCCIÓN DEL CENTRO DE BALANCEO.}

La ubicación del centro de balanceo se encuentra mediante la proyección de una línea desde el centro del contacto entre la rueda y la superficie de carrera del circuito a través del centro instantáneo de la vista frontal o posterior como se muestra en la figura 2 (líneas de color naranja). Cuando estas dos líneas se intersectan da como resultado el centro de balanceo de la masa suspendida del auto y no se encuentra ubicado necesariamente en la línea central del auto, sobre todo con una geometría de suspensión asimétrica como la de la figura 2 .

Figura 2. Ubicación del centro de balanceo.

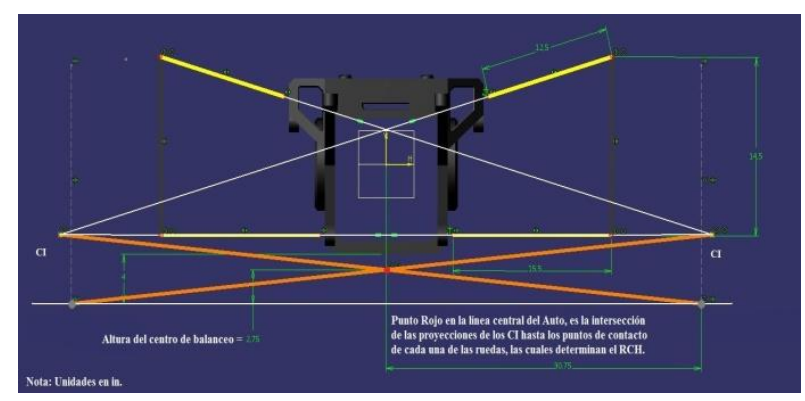

Fuente: Autores

La altura del centro de balanceo construida para la geometría de estudio está a 2.75 in del nivel de la superficie de carrera y es más bajo que los valores de altura de centros de balanceo para suspensiones posteriores (7 in-11 in), así que es un buen parámetro para el diseño final del sistema, ya que se reducirá el balanceo en la sección posterior del auto.

\section{RESTRICCIONES Y GRADOS DE LIBERTAD}

Para cualquier cuerpo moviéndose en el espacio respecto a otro cuerpo, su movimiento puede ser completamente definido por tres componentes de movimiento lineal y tres componentes de movimiento rotacional como se muestra en la figura 3 .

Se dice que un cuerpo solo tiene seis grados de libertad (D.O.F) de movimiento. Cualquier suspensión independiente permite solamente una trayectoria de movimiento con relación al chasis y a su vez ofrece cinco grados de restricción (D.O.R), que limita el movimiento en las cinco dimensiones restantes.

Figura 3. Grados de libertad y definición del movimiento de la suspensión.

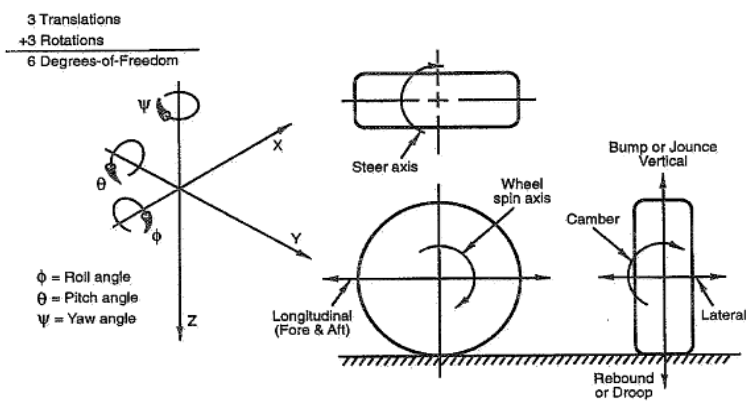

Fuente: MILLIKEN William, MILLIKEN Douglas, RACE CAR VEHICLE DYNAMICS, Editorial SAE, 1995.

Los componentes que se utilizan para el diseño final de la geometría de la suspensión son brazos de control con forma de A y 
como elementos de conexión se emplean articulaciones en los extremos como se muestra en la figura 4 , como las conexiones necesarias para esta configuración son cuatro, por lo tanto se obtienen cuatro grados de restricción dados por las cuatro articulaciones ubicadas en los extremos de anclaje en cada uno de los brazos de control y el conjunto resorte/amortiguador y el anclaje del brazo de control de la barra estabilizadora proporcionan una quinta restricción como se ve en la figura 5.

De esta forma queda restringido el movimiento lineal en $\mathrm{X}$ por los elementos de sujeción (tuercas hexagonales ranuradas) en el pivote estructural y por los anclajes de los elementos de fuerza (amortiguador y barra estabilizadora), en $\mathrm{Y}$ y $\mathrm{Z}$ el movimiento lineal queda restringido por el montaje de los brazos de control en un pivote estructural, y en cuanto los movimientos rotacionales quedan restringidos los pares cinemáticos en las direcciones de $\mathrm{Y}$ y $\mathrm{Z}$ permitiéndose solo la rotación en $\mathrm{X}$.

Figura 4. Grados de restricción dados por elementos de conexión.

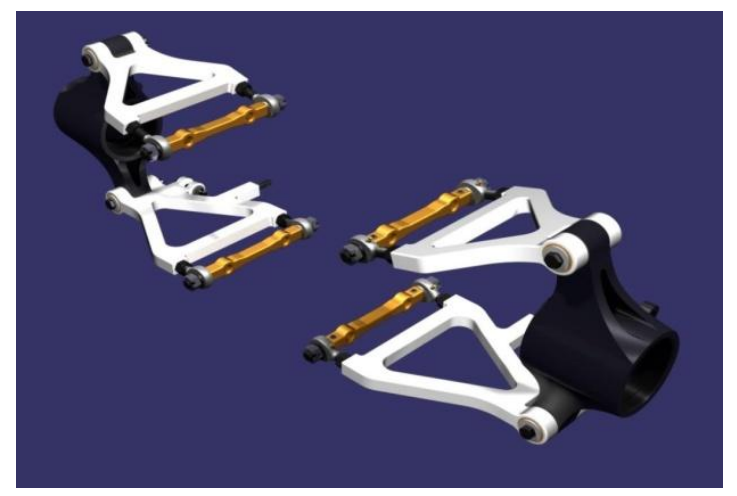

Fuente: Autores

El sistema que actualmente utilizan estos autos es una suspensión de tirantes, en donde hay un brazo que hace el trabajo de cinco conexiones (barra panhard), pero para hacerlo, debe ser fuerte a flexión y a torsión en las tres direcciones de rotación dándole así la rigidez característica de estos sistemas.
Figura 5. Grado de restricción dado por elementos de fuerza.

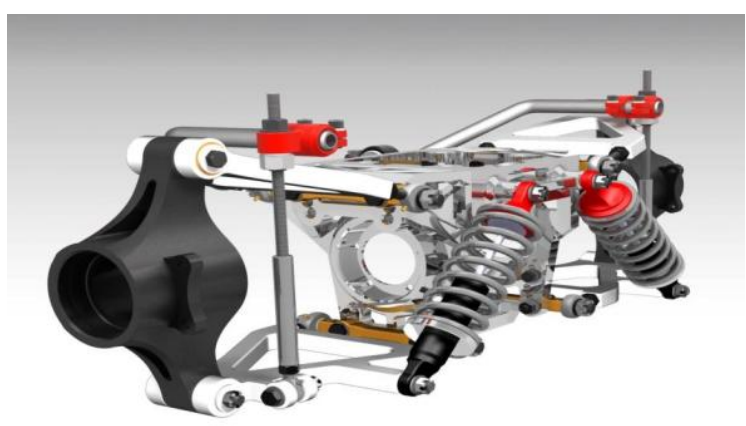

Fuente: Autores

El sistema multicuerpo de estudio se puede considerar como un conjunto de cuerpos individuales conectados entre sí mediante juntas cinemáticas y/o elementos de fuerza, permitiendo el movimiento relativo entre ellos. La geometría seleccionada para la suspensión es un mecanismo cerrado por lo que no tiene puntos o nodos de conexión con apertura y solo posee un grado de libertad dado por las articulaciones esféricas por que permiten la rotación sobre el pivote estructural sobre el cual van ensamblados los brazos de control (Figura 6).

Figura 6. Par cinemático de rotación

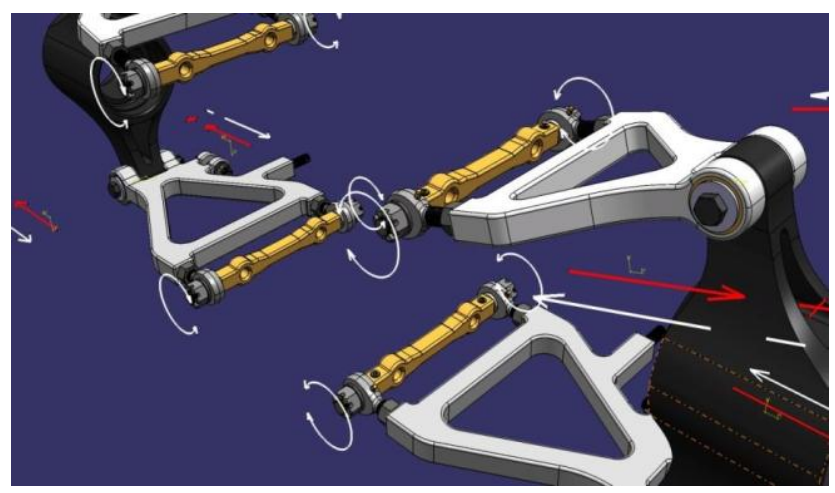

Fuente: Autores

\section{CÁLCULO Y ANÁLISIS}

Para el cálculo de los parámetros que definen la geometría de la suspensión seleccionada es necesario que sean ajustados lo mejor posible de acuerdo a la aplicación a 
la cual está destinada, según el análisis planteado anteriormente se recurre a un proceso iterativo, teniendo en cuenta como referencia los recorridos típicos para sistemas de suspensión para carreras, reduciendo así el parámetro scrub y el centro de balanceo, aunque cuando se habla de suspensiones independientes, la altura del centro de balanceo puede estar ubicado por debajo del nivel de la superficie de carrera de circuito. A continuación se relacionan en la Tabla 1 los recorridos típicos de sistemas de suspensión para autos según su aplicación.

Al evaluar el recorrido de la geometría seleccionada, el recorrido de la suspensión debe estar en la categoría de autos deportivos, autos de carrera y pequeños formula con una recorrido de in, el otro recorrido de la categoría no se selecciona por la razón de que como el chasis del auto está ubicado a una altura de 4 in medidos desde el suelo, con un recorrido de mayor amplitud el chasis impactaría con el suelo, a menos que en los componentes de la suspensión se coloquen una serie de topes que limitaran el recorrido.

Tabla 1. Recorridos típicos de sistemas de suspensión.

\begin{tabular}{c|c}
\hline Tipo de auto & Recorrido \\
\hline Autos todo terreno & \pm 12 in \\
\hline Autos de pasajeros & \pm 4 in \\
\hline $\begin{array}{c}\text { Autos Deportivos, autos de carreras y } \\
\text { pequeños autos de formula. Formula } \\
\text { SAE }\end{array}$ & \pm 2 in a \pm 4 in \\
\hline Autos efecto suelo, IRL y Formula 1 & \pm 0.5 in o menos \\
\hline
\end{tabular}

Fuente: MILLIKEN William, MILLIKEN Douglas, RACE CAR VEHICLE DYNAMICS, Editorial SAE, 1995.

A continuación en las Tablas 2 se detallan los resultados finales para la geometría que se piensa modelar con los parámetros estudiados scrub, altura del centro de balanceo y la variación del ángulo de caída.

Tabla 2. Constantes de Olley

\begin{tabular}{|cccc|}
\hline \multicolumn{4}{c}{ Cálculo de las constantes de Olley } \\
\hline P1 & Q1 & P2 & Q2 \\
0,015483871 & $-0,013548$ & 0,86180645 & 2,933419 \\
U1 & V1 & U2 & V2 \\
\hline $\mathbf{0 , 1 3 7 4 1 9 3 5 5}$ & $-0,1202419$ & 7,648532 & 26,0341 \\
\hline
\end{tabular}

Fuente: Autores

A medida que los brazos de la de una posición neutra, el centro de balanceo cambia de posición desplazándose hacia el nivel de la superficie de carrera del circuito y si la suspensión recorre una trayectoria vertical negativa la posición del centro de balanceo se aleja de la superficie de carrera del circuito, pero retornando siempre a su posición inicial de configuración o centro de balanceo estático, el tener un centro de balanceo bajo implica una menor transferencia de carga, por lo que se reduce el balanceo del auto cuando toma las curvas. El valor calculado es una buena referencia debido a que el centro de balanceo para suspensiones posteriores está comprendido entre 7 in y $11 \mathrm{in}$, por ende se puede decir que la transferencia de carga que se va a llevar a cabo en la suspensión posterior será menor.

Según las datos obtenidos la altura del centro de balanceo estático está ubicado en la línea central del auto a una altura de 2.756 in con un margen de error de $0.2 \%$ coincidiendo este valor con el obtenido por el método grafico en la figura 11, de esta forma queda comprobada la correcta ubicación de este parámetro para el diseño final de los componentes del sistema. Otro factor a tener en cuenta es la inclinación del brazo de control superior, si el brazo es muy corto y está muy inclinado, el desplazamiento dinámico del centro de balanceo es mayor, es decir, aunque mejora el ángulo de caída de la rueda más externa, 
contrarresta así la variación debida al balanceo del chasis, el ángulo de caída en la rueda interior puede ser desfavorable ocasionando un desgaste progresivo.

\section{DISTRIBUCIÓN DE PESO}

La distribución del peso es la forma más importante de cambiar la maniobrabilidad del auto. Cuando se hacen los ajustes de la distribución, hay que considerar los "pesos transitorios" que el auto experimenta mientras se lo está manejando.

Cuando se acelera el auto, el peso aumenta en la parte trasera, sobre las ruedas posteriores. Cuando hace el giro a la izquierda, el peso se incrementa sobre la vía derecha del auto debido a la fuerza centrífuga. El ajuste más útil del peso de un auto para condiciones de competencia es sobre la "vía frontal". El propósito de ajustar la distribución del peso en tal vía es balancear el auto entre la sección frontal y posterior mientras el auto pasa por la curva.

El tipo de pista sobre la cual se planea circular determina la distribución ideal del peso. Si las condiciones del trayecto requieren de acelerar al girar, entonces el peso será transferido al tren trasero mientras pasa por la curva, entonces, se puede agregar peso sobre el frente del auto para compensar la transferencia de peso cuando se está acelerando.

Cuando se maneja en pistas con el mismo número de curvas tanto a derecha como a izquierda, solo se debe ajustar el la distribución de peso en la sección frontal del auto. Sin embargo, hay dos ajustes más que pueden mejorar notablemente la maniobrabilidad si se está compitiendo en una pista oval o en una pista con predominio de curvas hacia la derecha o hacia la izquierda, en este caso la toma de curvas es siempre hacia la izquierda en los óvalos de la temporada de la NASCAR donde los ajustes adicionales se hacen incrementando el peso en la vía izquierda y alterando el cruce de peso.

El ajuste de la distribución del peso sobre las vía frontal y vía posterior del auto se calcula fácilmente mediante las ecuaciones 35 y 36. $\% R F=$ PESO TOTAL SOBRE AMBAS RUEDA FRONTALES O POSTERIORES
$/$ PESO TOTAL DEL AUTO X 100

\section{RESULTADOS}

Los elementos usados para el análisis de FEM de la geometría de estudio fueron elementos tridimensionales, específicamente tetraedros parabólicos por su excelente adaptación en geometrías complejas basadas en curvaturas y con un tamaño relativamente pequeño de 0.125 in con el fin de tener un mallado muy fino.

El cuerpo discretizado o el mallado que se observa en la figura 7 a menudo son creados con programas de generación de malla o programas procesadores integrados al software $\mathrm{CAD}$, en este caso el mallado se realizó mediante la herramienta Generative Structural Analysis del Software CATIA V5 R20.

Figura 7. Mallado del Modelo

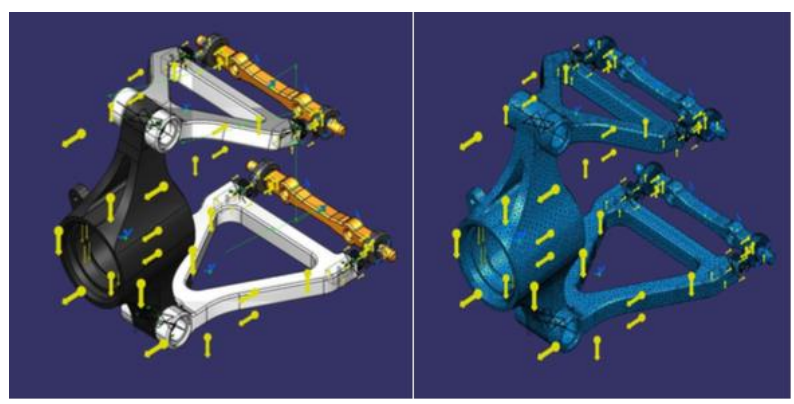

Fuente: Autores

Según los resultados obtenidos, la suspensión experimenta la mayor carga vertical en la sección derecha con una 
magnitud de 3659,95315 lbf e incide una fuerza lateral con magnitud de 3935,25 lbf. Evaluando los componentes mediante elementos finitos se obtuvieron los siguientes resultados.

El factor de seguridad para sistemas de suspensión de alto rendimiento oscila entre 2.5 y 3 , ya que las piezas que conforman el sistema de suspensión para estos autos están sometidas a cargas extremas (Fuerzas entre 3 y 4 G's) lo que implica el uso de un factor de seguridad alto con el cual asegurar la integridad de los componentes sin que sé que sufran una deformación permanente.

Para el analisis se empleo la seccion derecha del sistema con el fin de simplificar la estructura, Para el cálculo de los esfuerzos y del análisis por elementos finitos se utilizó la aleación de aluminio para fundición 355.0-T71, con la cual se fabricaran los brazos de control, manguetas y el modulo central.

Los resultados obtenidos para estos componentes por medio del software son los mostrados en la figura 6, donde los esfuerzos están comprendidos entre 1407, 6 psi y 8337,459 psi para la tensión (Zona de Color Amarillo y Naranja) y para la compresión están comprendidos entre 1876,139 psi y 10078,617 psi (Zona de Color Amarillo y Naranja).

Los esfuerzo calculados por el software para los brazos de control superiores en la compresión están comprendidos entre 685,759 psi (Zona Amarilla y verde) y 5033,144 psi (Zona Amarilla Naranja) mientras que para la tensión, los esfuerzo están comprendidos entre 499,447 psi (Zona Azul Verdosa) y 5198,695 psi (Zona Naranja).

El maximo esfuerzo calculado se encuentran en las rotulas esfericas de los brazos de control, las cuales son fabricadas con acero aleado cuyo esfuerzo permisible es de 104 ksi, aplicando de igual manera el factor de seguridad para este componente, se tiene una esfuerzo de diseño de 34666,66667 psi, el esfuerzo es resistido por el cuerpo roscado del elemento. Según el trazado de Von Misses de la figura 8 el valor es de 31887,597 psi y es menor en comparacion con el esfuerzo de diseño. Por lo tanto se afirma que la geometría estará siempre operando bajo las condiciones de diseño establecidas.

Figura 8. Análisis de FEM de ensamblaje de la sección derecha de la suspensión.

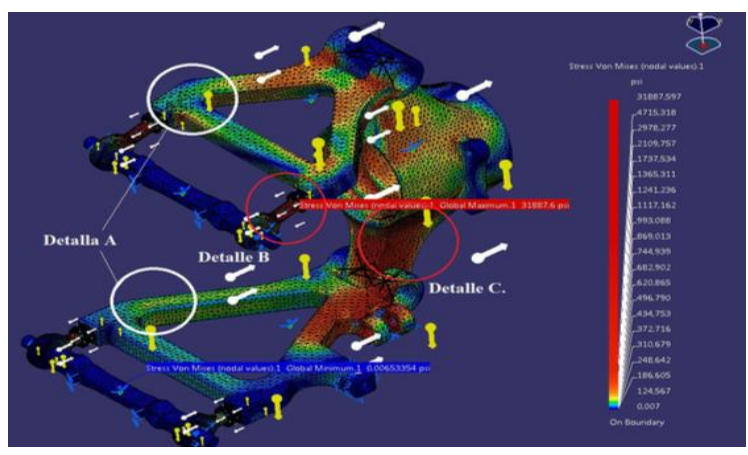

Fuente: Autores

Análisis del Módulo estructural central: Este es el componente principal, ya que todos los demás componentes van conectados a él, para el análisis en el software se empleó las mismas condiciones de operación al igual que en la sección derecha de la suspensión. Para la obtención de la geometría final que se observa en la figura se comenzó de un boceto preliminar, 
una vez definidas las características que se buscaban para ubicar la unidad motriz, se comenzó a realizar el modelo de la estructura en el software para poder así evaluar su comportamiento mecánico frente a las condiciones de operación.

De acuerdo a la magnitud de las fuerzas calculadas se fue corrigiendo la geometría según los resultados que se obtenían mediante el módulo de análisis estructural del software y a su vez optimizando cada sección del componente con el módulo Knowledgeware Product Engineering Optimizer con el fin de que la pieza estuviera dentro de los esfuerzo de diseño calculados anteriormente.

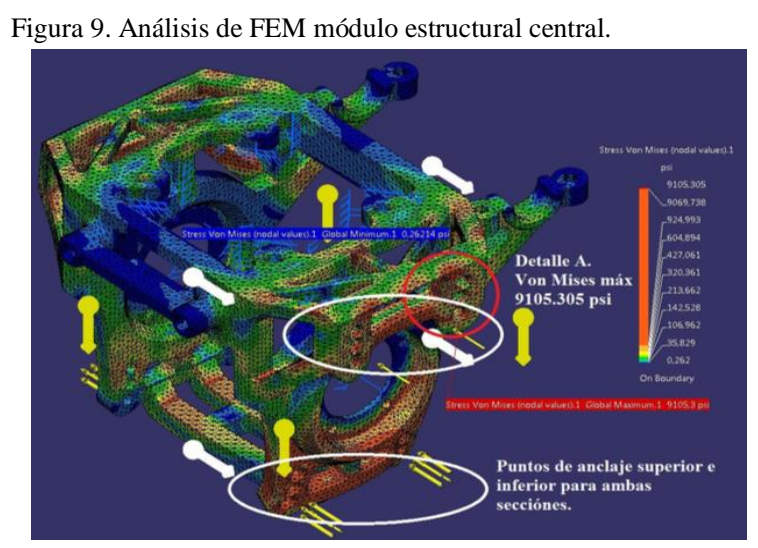

Fuente: Autores

Los puntos que se consideraron críticos para su análisis en el módulo central fueron los puntos de anclajes donde van ensamblados los brazos de control como se ve en la figura 45 con el área demarcada con elipses y los puntos de ensamblaje del conjunto coilover/amortiguador como se ve en el detalle $\mathrm{C}$ de la misma figura.. Los puntos para ensamble superior del conjunto coilover/amortiguador se evaluaron en la condición donde el elemento de fuerza se comprime ales su posición máxima, esta fase se logra con el máximo recorrido permitido por la suspensión el cual es +/- 2 in, por lo tanto las fuerzas calculadas para la dirección en el eje $Z$ es de 2585.25lbf y para el eje Y de 2505.28 lbf ya el elemento de fuerza está posicionado con una inclinación de 44.1o.

\section{CONCLUSIONES.}

Este proyecto proporciono la información necesaria para el desarrollo de sistemas de suspensión, dado que en el diseño de suspensiones no existe una geometría única, y esta puede variar como tantos diseñadores haya, se debe siempre conocer la aplicación y las características del auto para el cual se realizara el estudio.

Las referencias utilizadas y las herramientas computacionales han proporcionado el apoyo necesario para determinar las cargas a las cuales están sometidos los componentes del sistema de suspensión y los detalles del diseño en forma coherente con los cálculos obtenidos.

Aunque se obtuvo una excelente respuesta de los componentes que conforman la estructura principal del sistema de suspensión propuesto bajos condiciones de operación máximas aún falta realizar estudio de vibraciones.

La geometría del diseño propuesto de esta suspensión SLA posterior cuenta con las opciones de configuración deseadas, pero al reemplazar el sistema antiguo por el nuevo podría tener incidencia negativa sobre el sistema de suspensión frontal, por esta razón es importante realizar un análisis del sistema de suspensión frontal, con el fin de corregir la posición de los brazos de control frontales para que la transferencias de pesos llegue hacer lo más uniforme posible.

\section{BIBLIOGRAFÍA}


E. Bello, I. Ogedengbe, A. Osasona, A. Akinola. "Compromises in Vehicle Suspension Design" Journal of Mechanics Engineering and Automation. Vol. 2. 2012. pp. 441-445.

D. Cao, X. Son, M. Ahmadian. 'Editors' perspectives: road vehicle suspension design, dynamics, and control". Vehicle System Dynamics. Vol. 49. 2011. pp. 3-28.

COSTIN Michael, PHIPPS David, RACING AND SPORTS CAR CHASSIS DESIGN, Editorial B.T. BATSFORD. 1974, Chapter VII Suspension Principles, Chapter VIII Suspension Practice and Types, Pg. 2648, Pg. 49-55, Pg. 69-88.

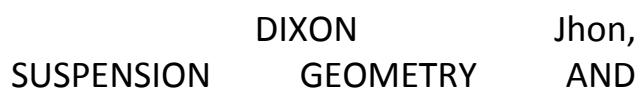
COMPUTATION, Editorial Wiley, 2009, Chapter 7 camber and Scrub, Chapter 8 Roll Center, Chapter 10 Pitch Geometry, Chapter 12 Double arm Suspension, Pg. 143-156, Pg. 157-177, Pg 189- 193.

B. Hall. "Suspension systems and components". An introduction to Modern Vehicle Design. J. Happian (editor). First Edition. Ed. Butterworth Heinemann. Linacre House, Jordan Hill. Oxford, England. 2002. pp. 277-326.

JAZAR Reza, VEHICLE DYNAMICS Theory and Application, Editorial Springer, 2008, Chapter 8 Suspension Mechanisms, Chapter 10 Vehicle Planar Dynamics, Chapter 11 Vehicle Dynamics, Pg. 455-497, Pg 583635, Pg 665-693.
MILLIKEN William, MILLIKEN Douglas, RACE CAR VEHICLE DYNAMICS, Editorial SAE, 1995, Chapter 17. Suspension Geometry. Pg. 608-621

PUHN Fred, HOW TO MAKE YOUR CAR HANDLE, Editorial H.P. Books, 1976, Chapter 2 General information on handling, Chapter 3 Suspension Tuning, Pg. 10-49, Pg 51109.

STANIFORTH Allan,
COMPETITION CAR SUSPENSION,
Editorial Hayes Publishing, 1999,
Chapter III Location and Chapter IV The
Winning Package, Pg. 57-92, Pg. 93-120.

